\title{
Human face detection and recognition using contour generation and matching algorithm
}

\author{
Arulananth T $\mathbf{S}^{1}$, Baskar $\mathbf{M}^{2}$, Sateesh $\mathbf{R}^{3}$ \\ ${ }^{1}$ Professor, Department of Electronics and Communication Engineering, MLR Institute of Technology, India \\ ${ }^{2}$ Professor, Department of Information Technology, MLR Institute of Technology, India \\ ${ }^{3}$ Assistant Professor, Department of Electronics and Communication Engineering, MLR Institute of Technology, India
}

\begin{tabular}{l} 
Article Info \\
\hline Article history: \\
Received Jan 26, 2019 \\
Revised Apr 30, 2019 \\
Accepted May 25, 2019 \\
\hline
\end{tabular}

Keywords:

Contour matching

Face detection

Face monitoring

Face recognition

Geometric contours

\begin{abstract}
Face detection, monitoring of human face and recognition is a high-quality venture in safety, surveillance and regulation enforcement systems with greater accuracy. There are many motives at the back of in this trouble. Which include function of the human face; pose version, negative environmental conditions, terrible lightning and picture tilt etc,. So we must locate a few feasible solutions to clear up those issues by means of imposing a brand new system. Our proposed venture might be improving the issues on Face detection, monitoring of human face and recognition issues.
\end{abstract} All rights reserved.

\section{Corresponding Author:}

Arulananth T S,

Department of Electronics and Communication Engineering,

MLR Institute of Technology, India.

Email: arulanandh.ts@gmail.com

\section{INTRODUCTION}

Face detection, monitoring of human face and recognition is an amazing project in safety, surveillance and law enforcement structures with greater accuracy [1]. There are many reasons at the back of on this problem. Inclusive of role of the human face, pose variant, negative environmental conditions, terrible lightning and picture tilt and so forth. So we ought to find a few possible answers to clear up these troubles through imposing a brand new gadget. First we can get the frames camera or web cam. At the same time, a detector analyzes the frames to find a face and highlight it within the panel. Face Tracker is shown in motion within the pictures on the top of the web page [2,3].

The detection algorithm is executed speedily while there is a face present in the image (it will be around 40ms), however may additionally take substantially longer to decide there may be no face (as plenty as $200 \mathrm{~ms}$ ). Essential aspects of the coding are locating ways to speed up the detection, and making sure that lengthy detection processing don't sluggish down the relaxation of this system (in particular, the rapid rendering of successive images onto the panel) [4]. Detection is finished with the aid of a contours matching algorithm, pre-educated to find facial features (whilst regarded the front-on). The classifier's education calls for a remarkable deal of time.

The subsequent processing will increase to understand the tracked face. The difference between face detection and reputation is that popularity returns a call for the face. The control software program for the launcher ought to then decide whether the individual is a chum or for before firing a missile. Reorganization is applied by connected thing Operators [5]. Contour matching set of rules used for identification of faces. The feasibility of the usage of contour matching for human face has been identified and it is presented through experimental research. The benefit of the use of contour matching is that the structure of the face is 
strongly represented in its description along with its algorithmic and computational simplicity that makes it suitable for hardware implementation [6]. The input contour is matched with registered contour the usage of simple matching algorithms. The matching results received show that with a small diploma of panning and tilting, the outcomes are pretty exact.

\section{LITERATURE SURVEY}

SHU Chang \& et al., 2011, in this paper the Histogram of Oriented Gradient has been effectively implemented in lots of research fields with first-rate performance in particular in pedestrian detection. But, the method has not often been implemented to face popularity [1]. Tong Chen \& et al., 2014, on this paper, they advise a non-touch detection method that makes use of a physiological signal and this technique permits dimension of a physiological function at standoff distances [2].

TAN Chunlin \& et al., 2010, on this paper, the Scale Invariant Feature rework (SIFT) characteristic descriptor is invariant to photo scale and location, and is robust to affine transformations and modifications in illumination, so it's far a effective descriptor used in many packages, such as item recognition, video monitoring, and gesture popularity [3]. YI-CHEN et al., 2015, in this work, to recognize the human in unconstrained video, one has to explore the identity information in multiple frames and the accompanying dynamic signature. These identity cues include face, body, and motion. Our approach is based on videodictionaries and it is a generalization of sparse representation and dictionaries for still images [4].

Siyang Yan \& et al., 2016, they are proposes an automated and robust method to come across human faces detection [5]. MA Yong \& et al., 2003, in this paper, describe locating multi-view faces an images with a complex background remains a challenging problem for face detection. An integrated method for real-time multi-view face detection and pose estimation is presented. A simple-to-complex and coarse-tofine view-based detector architecture has been designed to detect multi view faces and estimate their poses efficiently. Both the pose estimators and the view-based face/non-face detectors are trained by a costsensitive AdaBoost algorithm. AdaBoost algorithm is implemented to improve the generalization ability [6].

Gita Indah Hapsari \& et al, 2017, this paper proposed a portable, real time, and wearable product. Raspberry Pi supports portability that affects the delay and computing speed of face recognition algorithms. It needs 3 seconds for one-face recognized in range 0.25 until 1.5 meters from the camera, until the sound and information are generated. It needs is me 5 second for two faces recognized and 10 seconds for 3 faces recognized by a system in the same range between face and the camera. The accuracy reaches $91.67 \%$ for the up-right position face but for other position the accuracy is only $18 \%$ until $32 \%$ [7].

Edy Winarno \& et al, 2017, the proposed half-join method is a face images joining model, which is called asymmetrical half-join. In asymmetrical half-join method, a RoI (region of interest) of face image from left and right lenses are provided based on axis center of each eye in eye detection [8]. Muhammad Fachrurrozi \& et al, 2018, multi-face image search using Content Based Image Retrieval (CBIR) method. CBIR performs image search by image feature itself. Based on real time trial results, the accuracy value obtained is $61.64 \%$ [9].

I Nyoman Gede Arya Astawa \& et al, 2018, in this experiment, intensity normality method in one of the color space channel and tested the faces using Android based have been developed. The faces multi image datasets came from social media, mobile phone and digital camera. In this experiment, the color space $\mathrm{YCbCr}$ percentage value with the image initial value detection before processing are $67.15 \%, 75.00 \%$, and $64.58 \%$ have been reached [10].

Shivkaran Ravidas \& et al, 2018, the aim of this paper is to detect multi-view faces using deep convolutional neural network (DCNN). Implementation, detection and retrieval of faces will be obtained with the help of direct visual matching technology. Further, the probabilistic measure of the similarity of the face images will be done using Bayesian analysis [11].

In this work we propose a new approach for detecting the boundaries of lesion in mammography images based on region growing algorithm without using the threshold, the proposed method requires an initial rectangle surrounding the lesion selected manually by the radiologist (Region Of Interest), where the region growing algorithm applies on lines segments that attach each pixel of this rectangle with the seed point, such as the ends (seeds) of each line segment grow in a direction towards one another [12].

\section{METHODOLOGY}

This block diagram can be explained through three main steps they are:

a. Image Processing and Normalization process

b. Contour generation

c. Image Matching algorithm 


\subsection{Image Processing and Normalization Process}

From the Figure 1, the captured images are divided into two jointly one of a kind sets: the trained set and the test set. The trained set is used to start and prepare the device to identify subjective pix and to highquality composition the algorithm parameters. The take a look at set is the set of photographs that is used to evaluate the performance of the machine after schooling is completed [13]. The captured images are preprocessed to improve the popularity overall performance. After the pre-processing stage, all of the new images ought to an identical in dimension, so after cropping the face from the whole image, those new snap shots are resized to $150 \times 112$ pixels. Any other difficulty particular to faces, is that of facial features, complexion and whether or not the concern is carrying glasses or has a moustache and/or a beard. The device should be able to identify faces whether or not they may be smiling, sad, sporting glasses, and not wearing glasses. For this reason its miles essential to apply a trained face database that includes different pictures of identical challenge. The photographs should be preprocessed to lead those to suitable for reputation purposes. This usually consists of the following responsibilities:

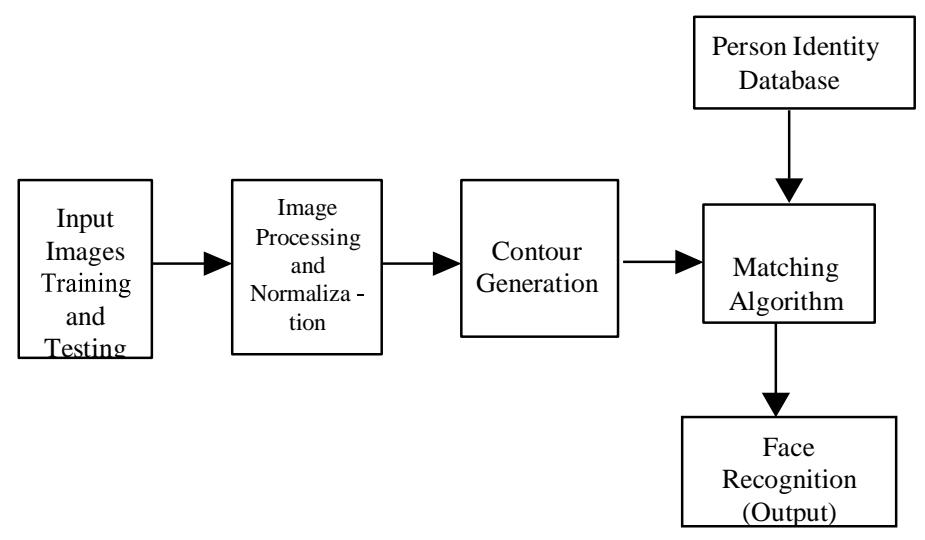

Figure 1. Block diagram of proposed algorithm

\subsection{Histogram Equalization}

Histogram equalization is achieved which enhances the assessment of photographs with the aid of transforming the values in a depth photograph in order that the histogram of the output picture about matches a specified histogram.

\subsection{Noise removal}

This step is used to estimate and remove any noise if gift within the image.

\subsection{Normalization}

This is used to compensate for any illumination versions or relative sizes among two sets of faces. That is accomplished the usage of pixel cost normalization. In this stage the imply pixel fee of all of the pixels within the image is calculated. Also the usual deviation of pixel values is calculated. The usage of those values normalize the pixel values of the picture by the usage of the subsequent components Pixel price normalized image $=($ Blurred photograph pixel - Pixel suggest $) /$ fashionable deviation.

\subsection{Contour generation}

Middle of the system in which the contour of a face is generated from the picture. The whole human face is considered as a contour map, with the areas of steady gray stage brightness together with this by means of the contour traces. For this reason contour lines for a given face may be generated.

a. Getting to know face look fashions and models for realtime observable motion estimation \& clustering

b. Studying Gaussian mixture primarily based shade fashions for equally monitoring pores and skin tone items and multi-color based forefront and historical past segmentation and tracking.

c. Gaining knowledge of an adaptive temporal coloration version to address extreme lights adjustments.

The primary difficulty in modeling shade robustly is the shade fidelity problem which arises because of version in shade values delivered about via lighting adjustments. Information fusion in object detection and monitoring the usage of both motion and color cues was used to result in the specified consistency in face tracking. The device is able to perform face detection and tracking in the following way. 
d. Actual-time detection and tracking of moving faces in cluttered scenes.

e. Robust monitoring of multiple shifting faces.

f. Robust monitoring below changes in lights, scale and photograph decision,

g. Robust tracking beneath "facial distortions" including spectacle, facial hair and hair-fashion modifications.

Related to face detection and tracking, we also addressed the problem of actual-time head pose estimation which is important in monitoring transferring faces across perspectives [14]. We introduced a composite Gabor wavelet transform as a representation scheme for capturing pose changes. We derived a pose Eigen-area primarily based at the essential additives evaluation to represent and interpret the distribution of pose modifications from continuous sequences of face rotation intensive [15].

\subsection{Different Image Matching Algorithms}

Four image matching strategies are very generally used. A) Blob Detection Technique B) Template Matching C) SURF feature extraction and D) SIFT. In our work preferably use Template Matching strategies because Template matching is a 'brute-force' algorithm for object recognition.

\section{RESULT AND DISCUSSIONS}

\subsection{Case i: Image Position Detection}

The exclusive role of a human face had been captured and presented to the algorithm for role detection. Each role turned into modulated with a selected constellation, noise changed into added and filtered for actual time consequences and the detected role became as compared with the real. The modulation of position facts turned into accomplished the usage of QAM-256 scheme [16]. The captured input positions, matching algorithm all through runtime and the categorized output is proven in Figure 2 to determine 3 .

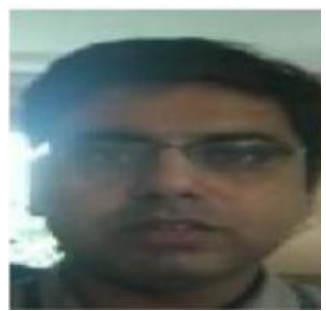

(a) Face Position 1

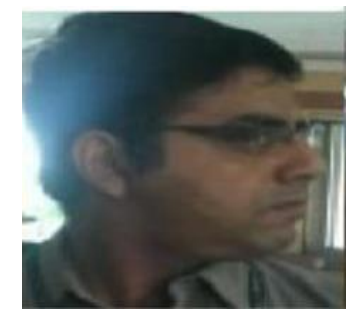

(b) Face Position 2

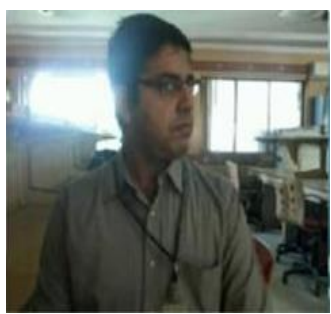

(c) Face Position 3

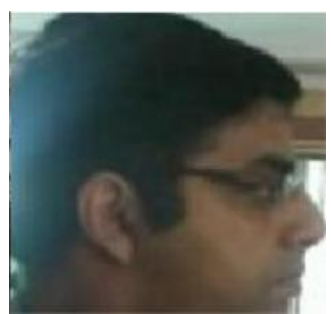

(d) Face Position 4

Figure 2. Shows the Various Captured Sample Input Image for Position Detection

\subsection{Case ii: Privacy Preservation in Video Images}

The textual content turned into hidden inside the video and to protect the data privateness, slicing of video picture has been done. The input frame, sliced frames and the matched photos (i.e. where the question text matched with the hidden text) are proven in Figure 3(a) to parent 3(c). The video frames available for public storage corresponds to the sliced frames and as a result privacy protection is finished. A pattern of the three snap shots, the equal textual content turned into hidden and the corresponding pictures on my own had been correctly recovered.

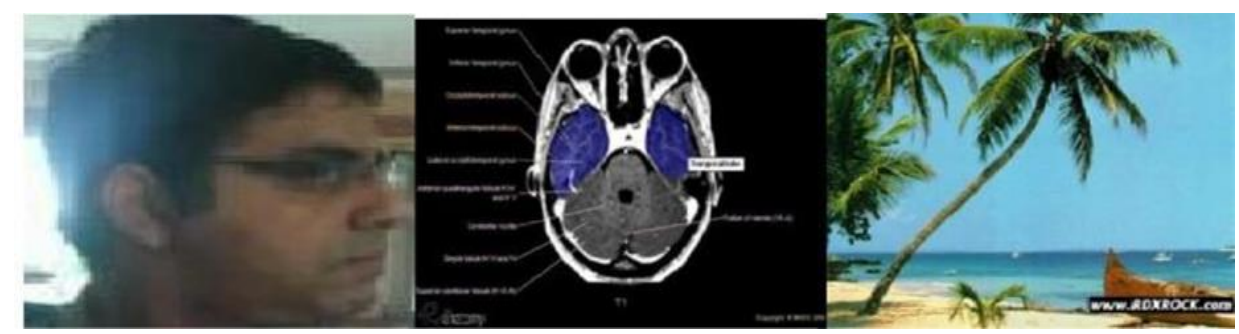

Figure 3(a). Input Images 


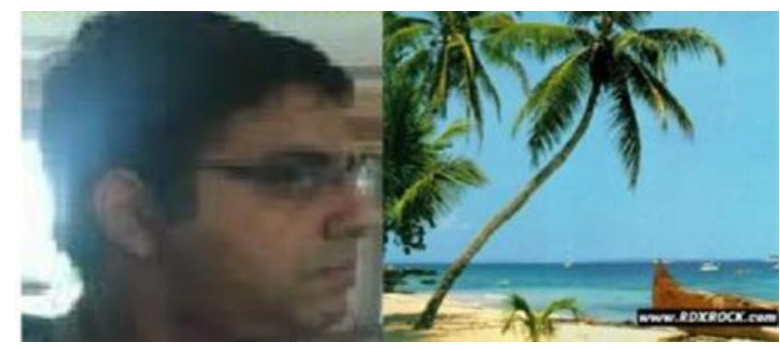

Figure 3(b). Matched output images from the input raw images

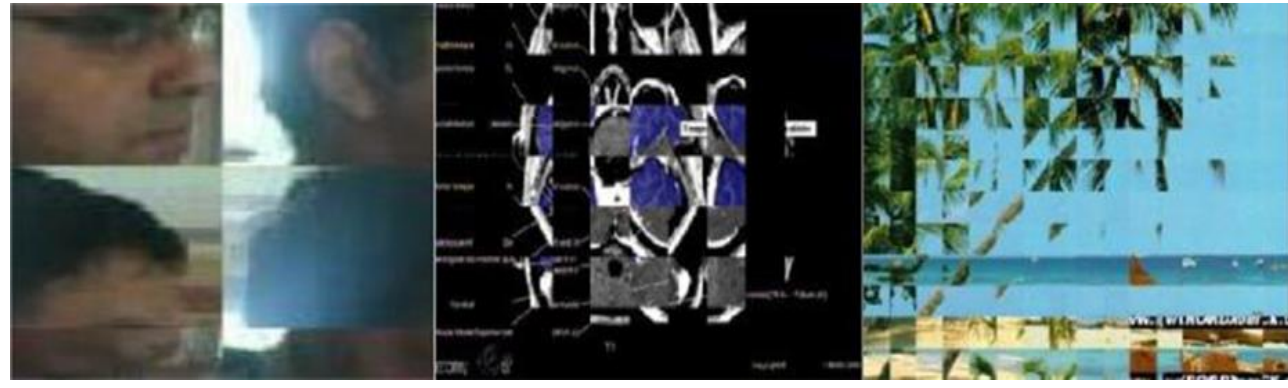

Figure 3(c). Sampled Slice Images with poor conditions

An image-based tracking system can detect the correct position of the moving human face images even poor lightning. Tracking accuracy, processing time and applicability to poor environment is the identical in this method. Consequently, this research improves the performance of the image tracking system to be simplified and not required a separate tracking system. The new algorithm has been implemented is also able to successfully detect the fall event within $255 \mathrm{~ms}$. Once the corresponding feature is verified by the current state, it can proceed to next state; otherwise, the system resets to the initial state and waiting for the appearance of another feature sequence. Moreover, a distinguished performance up to $92 \%$ on the sensitivity and $99.75 \%$ on the specificity is obtained with a set of 450 test activities.

\section{CONCLUSION}

Use of our proposed method we can detect and track the human face with higher efficiency is $10 \%$ better than the existing methods of image detection and tracking techniques. In terms of face recognition also it will provide better response over the available methods. Also this technique reduces the delay of response considerably. Because of these advantages it will be useful in Security systems, Classification of images, Image enhancement, Automatic image rotation, Portrait / passport photography, Law Enforcement Surveillance etc. This work is not a location specific and it can be used by everywhere.

\section{ACKNOWLEDGEMENTS}

The authors would like to thank the management of MLR Institute of Technology, KCG College of Technology and our faculty friends for proving this best opportunity.

\section{REFERENCES}

[1] S. Chang, et al., "Histogram of the Oriented Gradient for Face Recognition," vol. 16, pp. 216-224, Apr 2011.

[2] T. T. Chen, et al., "Detection of Psychological Stress Using a Hyper spectral Imaging," vol. 5, pp. 391-405, 2014.

[3] T. Chunlin, et al., "SWF-SIFT Approach for Infrared Face Recognition," vol. 15, pp. 357-362, 2010.

[4] Y. Chen, et al., "Dictionary-Based Face and Person Recognition from Unconstrained Video," vol. 3, pp 1783-1798, 2015.

[5] S. Yan, et al., "A Face Detection Method Combining Improved AdaBoost Algorithm and Template Matching in Video Sequence," 8th International Conference on Intelligent Human-Machine Systems and Cybernetics (IHMSC), vol. 02, pp. 231-235, 2016.

[6] Y. Ma and X. Ding, "Robust real-time face detection based on cost-sensitive AdaBoost method," vol. 2, pp. 465$468,2003$.

Human face detection and recognition using contour generation and matching algorithm (Arulananth T S) 
[7] P. I. Rani and K. Muneeswaran, "Robust real time face detection automatically from video sequence based on Haar features," International Conference on Communication and Network Technologies, pp. 276-280, 2014.

[8] G. I. Hapsari, et al., "Face recognition smart cane using haar-like features and eigenfaces," TELKOMNIKA Telecommunication, Computing, Electronics and Control, vol. 17.

[9] E. Winarno, et al., "Asymmetrical Half-join Method on Dual Vision Face Recognition," International Lournal of Electrical and Computer Engineering (IJECE), vol, 7.

[10] M. Fachrurrozi, et al., "Real-time Multi-object Face Recognition Using Content Based Image Retrieval (CBIR)," International Lournal of Electrical and Computer Engineering (IJECE), vol. 8.

[11] I. N. G. A. Astawa, et al., "The Impact of Color Space and Intensity Normalization to Face Detection Performance," TELKOMNIKA Telecommunication, Computing, Electronics and Control, vol. 15.

[12] M. Rmili, et al., "A New Approach to the Detection of Mammogram Boundary," International Lournal of Electrical and Computer Engineering (IJECE), vol. 8.

[13] S. Ravidas and M. A. Ansari, "Deep learning for pose-invariant face detection in unconstrained environment," International Lournal of Electrical and Computer Engineering (IJECE), vol. 9.

[14] S. Gupta, et al., "Analysis of training parameters for classifiers based on Haar-like features to detect human faces International," Conference on Image Information Processing, pp. 566-571, 2011.

[15] G. Papandreou, "Multigrid Geometric Active Contour Models," IEEE transactions on image processing, vol. 16, pp. 229-240, Jan 2007.

[16] T. S. Arulananth, et al., "Video Image Detection and Tracking Using PCA And SVM KNN Classifier with Hardware Implementation and Design," International Journal of Advanced Engineering Technology, vol. 7, pp. 6670, 2015.

\section{BIOGRAPHIES OF AUTHORS}

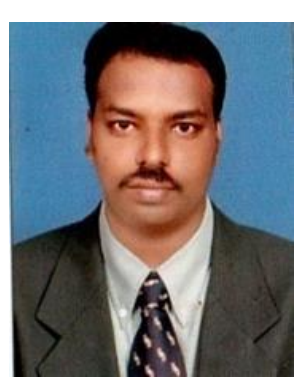

Dr. Arulananth T S has received B.E degree from Fx. Engineering College Thirunelveli, Tamilnadu, India in the year of 2004, M.Tech from Dr. M.G.R. Educational and Research Institute University Chennai-95, Tamilnadu, India in the year of 2007. I have completed Ph.D degree in Dr. M.G.R. Educational and Research Institute University, Chennai-95, Tamilnadu, India in the year of 2016. Currently he is working as a Professor \& I/C HOD in the Electronics and Communication Engineering in MLR Institute of Technology, Hyderabad-43, India. His research interests include image processing, Embedded Systems and communication systems. He was published three books with the titles of Microprocessors and Microcontrollers, Principle of Digital Signal Processing \& Discrete Time Systems and Signal Processing. He is a Life time Professional body member of ISTE, ISRD, IRED, IACSET, IAENG and UACEE and reviewer of some of the reputed International journals. And act as an advisory committee member for the two international conferences in India. Also his research/study work has been published in more than twenty five international / National journals and conference proceeding. He was awarded by Institute of self Reliance, Bhuveneshwer, Odissa, India by November 2017 for his wonderful contributions in Electronics and Communication Engineering. He was visited Cambridge University in United Kingdom in the beginning of 2018 for the presentation of his research work. Recently he was certified as internatuional engineering educator by IUCEE on active learning strategy.

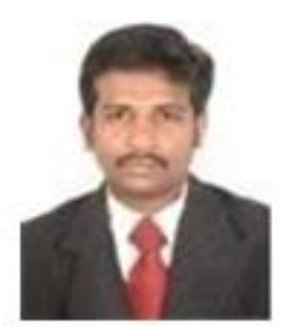

Mr. Sateesh R has received B.E degree from Sri Raja Rajeshwari Engineering College, Karepally in the year of 2010, M.Tech from ADAMS Engineering College, and Palvoncha India in the year of 2013. Currently he is working as a Assistant Professor in the Electronics and Communication Engineering in MLR Institute of Technology, Hyderabad-43, India. His researches interests include image processing, Embedded Systems and Systems \& Signal Processing.

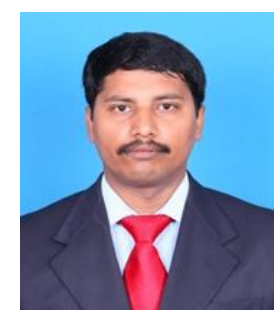

Dr. M. Baskar received B.E. Computer Science and Engineering from Anna University, Chennai, and M.Tech. Information Technology from Sathyabama University, Chennai, Ph.D., (I\&C) from Anna University, Chennai. His Area of research interest includes Computer Networks and Security, Parallel and Distributed Systems, Image Processing, Big Data, Machine Learning and IoT. He is published 18 Research Article in reputed International Journals and 9 Article in International Conferences. He is acting as a reviewer in Cluster Computing, Journal of Web Engineering, Multimedia Tools and Applications, Neural Processing Letters and Concurrency and Computation: Practice and Experience. 